\title{
Chemical and Bio Synthesis use-cases: Edible Polymers, Carbon Capturing, and CBD Isolate Phytocannabinoids
}

\author{
Andrew M. K. Nassief ${ }^{1}$ \\ ${ }^{1}$ Affiliation not available
}

February 26, 2021

\begin{abstract}
Computationally artificially derived chemical synthesis and multistep preparation processes can have a variety of use-cases. The idea of utilizing advanced computational complexity, bleu-score computational ranking type systems, and isolating different variable data is integrated in the synthesis preparation processes. Some use-cases presented include: edible polymers and the field study of printable foods, synthetic carbon capturing polymers and biomasses, and CBD Isolate for phytocannabinoids. Synthetic chemistry can tackle a variety of issues from food shortage to the opioid crisis. A computational scoring system can help with elimination reactions and non or organic reactionary mechanisms. Also, accuracy through validation-scoring and forms of molecular mechanics is crucial in the experimentation process. The same applies to various forms of variances, scattering and composition accuracy. The easiest way to demonstrate some of these concepts is through the analysis of said systems for these usecase varieties.
\end{abstract}

Author's Note: In regards to a computationally derived pipeline for synthetic processes.

\section{Hosted file}

Chemical Synthesis 2.pdf available at https://authorea.com/users/289895/articles/510866chemical-and-bio-synthesis-use-cases-edible-polymers-carbon-capturing-and-cbd-isolatephytocannabinoids 